\title{
Real-time laboratory surveillance of Dengue antigen (NS1) - Best proposed early warning and response system for Dengue in Sri Lanka? - Results from a pilot study
}

\author{
Lasantha Ranwala $^{1}$, Yudhisteera Wedisinghe ${ }^{2}$, Sathira Perera ${ }^{1}$, Deepal Jayamanne ${ }^{3}$ \\ (Index words: early warning and response system, NS1 surveillance, real-time surveillance, real-time biosurveillance, \\ Dengue, Dengue notifications)
}

\begin{abstract}
Introduction: During the recent past, dengue fever and associated complications have been the most important concern for health policy makers of Sri Lanka. The current notification system has considerable inevitable delays in preventive measures.

Objectives: Implementing a laboratory-based real-time antigen(NS1) surveillance system for notification coupled with a rapid preventive response within the Colombo district as a pilot project and compare the notifications with existing national surveillance systems.

Methods: An online notification platform was established with a centralized database. Seven main laboratories of the private sector linked with the notification system, where they can create new notifications at the central database, whenever the NS1 test detected as positive. Relevant Medical Officers of Health should update action implemented to complete the response process. A dashboard was designed to visualize each notification and its status with a predefined color code.

Results: Patients from 14 Medical Officer of Health (MOH) areas out of 15 were captured. The immediate preventive response was recorded from the field preventive staff for $90 \%$ of the reportings. Almost all attended patients have given health advice on awareness, prevention, and source reduction through premise inspection by trained field staff with $24 \mathrm{hrs}$ of notification.

Conclusions: Salient features of the novel system are notification of antigen-positive patients, the rapidity of notification(real-time) and response, user-friendliness, access to multiple stakeholders simultaneously without
\end{abstract}

data duplication, early involvement of the field staff, the ability to trace the cases using checklists and a colorcoding system from a dashboard.

\section{Introduction}

Dengue is the major vector borne disease in Sri Lanka where it has become hyper endemic in Sri Lanka. The year 2017 showed highest recorded number of cases ever in Sri Lanka history reporting more than 180,000 confirmed cases with an overall incidence around 865 per 100,000 population [1] and the majority were males (55\%) and aged between 25 to 49 years of age (37\%)[2]. Number of deaths were 440 with a case fatality ratio (CFR) of 0.24 .

Patient reporting shows two peaks where it correlates with seasonal (monsoonal) rain pattern and the geographical areas of the country. The peak from the $23^{\text {rd }}$ to $37^{\text {th }}$ weeks correlates with the south west monsoonal rain and wet zone of the country where the other peak correspond with the north eastern monsoonal rain and dry zone of the country [1,3]. During the year 2017 the excessive patient load was mainly due to change of predominant serotype to DENV2 (which the vulnerable population were not immune) from DENV4 and DENV1 which were circulating for more than 10 years in the country[4-6]. Western province of Sri Lanka had $41.4 \%$ of the total caseload with a case fatality ratio of 0.27 during 2017 preceded by 0.19 during 2016.

Currently available surveillance systems (routine, sentinel site and special) had limited potential of generating

Ceylon Medical Journal 2021; 66: 121-128

DOI: http://doi.org/10.4038/cmj.v66i3.9490

${ }^{1}$ Regional Directorate of Health Services, Colombo District, Colombo 10, Sri Lanka, ${ }^{2}$ Provincial Directorate of Health Services, Western Province, Colombo 10, Sri Lanka, ${ }^{3}$ Ministry of Health, Colombo 10, Sri Lanka, ${ }^{4}$ Department of Public Health, Faculty of Medicine, University of Kelaniya, Sri Lanka.

Correspondence: BDWJ, e-mail: <bdw.jayamanne@kIn.ac.lk>. Received 09 November 2019 and revised version 10 June 2021 accepted 15 June 2021

This is an open-access article distributed under the terms of the Creative Commons Attribution License, which permits unrestricted use, distribution, and reproduction in any medium, provided the original author and source are credited. 
effective early warning signals in respective localities this massive outbreak before reaches it peak of 10,684 patients per week during the $29^{\text {th }}$ week of 2017 [1] which is more than 4 folds greater than the national historical 5 year mean of highest recorded per week island wide. It has been challenging how the epidemic could be effectively controlled. Current paper based web based notification system where the notification is done on clinical suspicion has a considerable delays in investigating and preventive actions for reported cases [7].

The antigen (NS1) testing for Dengue with or without antibody (IgM) testing for definite diagnosis of the disease at an early stage of febrile patients has become a common practise in Sri Lankan private sector, especially in urban areas where dengue is more common $[6,8,9]$. Most of the private laboratories do the test by rapid test kit where few do ELISA. With the advancement of technology, both commercially available rapid test kits have achieved significant improvement of sensitivity and specificity of screening of fever patients without a focus $[10,11]$. Provincial department of health (PDHS) Western province has introduced real time laboratory surveillance of Dengue NS1 antigen detection as a new strategy to generate an early signal for potential outbreak of dengue as an alternative parallel early warning and response system (EWARS). This pilot project results have been compared with existing surveillance systems.

\section{Materials and Methods Development}

In 2013 an eHealth steering committee was established at the Western Provincial Department of Health along with Colombo, Gampaha and Kalutara regional directorates with the vision of establishing a sustainable ICT infrastructure, improvement of end user ICT literacy and service enhancement. This committee consisted of administrators, medical officers trained in health informatics as well as supporting staff. A strategic plan with roadmap to achieve key milestones was drafted after several round table discussions. The project was started after obtaining necessary administrative and operational approvals from the relevant health departments with funding from the Western Provincial Health Department of Sri Lanka.

The software for the system was developed as an inhouse product of the provincial health department using agile development methodology to accommodate requirement of early warning and response (EWARS) for Dengue as a module of Western provincial departmental health information system .

This was developed using PHP [12], MySQL [13] with JavaScripts, Cascaded Style Sheets (CSS) [14] and Hypertext Markup Language (HTML) [15] under General Public License (GPL) [16].Versions of the software were managed by Gitlab [17] where the versioning were deposited on a dedicated server of the department as well as on a remote server[18].

\section{Implementation}

The final tested version of the web based real time electronic antigen positivity notification system was implemented in 2016. Required training to handle the software was given to relevant staff members and necessary printed material (guides) and helpline was established for over the phone support. Seven major private laboratories were connected with the central database and a notification dashboard at the provincial epidemiology unit of the Western province with close monitoring. The central database also connected with the respective health division (Medical officer of Health$\mathrm{MOH}$ ) areas where the grass root level preventive measures are taken.

\section{Process}

Notification process initiated from the respective laboratory when a tested blood sample became positive for Dengue NS1 antigen test. With the patient's informed consent for notification, the relevant demographic data (contact no, address,etc) also collected and patient data is fed to the system by the responsible trained laboratory staff member who has credentials to log on. The assigned dashboard operator at the central level who gets notifications of all the laboratories, organises and directed the notifications to the respective $\mathrm{MOH}$ divisions on the sametime with the available details.

Whenever a MOH areas is assigned a new notification, it appears at the $\mathrm{MOH}$ users dashboard as "new notices". When the MOH level preventive staff notified the new case, it will prompted for pending action and following preventive action (response) they have to mark them as "completed". All the actions are colour coded to improve user interactions and response (White - New notification, Red - MOH to be noted, Yellow - pending for action, Green-actions completed, Grey - unidentified). Different customised reports at different user levels can be generated by the system accordingly.

Directives were given to take mandatory preventive measures including health education, source reduction with or without fumigation as instructed for the index and around $300 \mathrm{~m}$ radius around the index premises within 48 hours of notification to the system. Trained staff on prevention (called as rapid response team for Dengue) was managed centrally at the provincial office were mobilised in addition to the field staff of the respective $\mathrm{MOH}$ area. The system was updated as "completed" immediately at the $\mathrm{MOH}$ level as the feedback.

\section{Data analysis}

Data were analysed during the $15^{\text {th }}$ to $24^{\text {th }}$ weeks of 2016 from the system compared with the National routine 
surveillance (by weekly return of communicable diseases - WRCD) data and sentinel surveillance data (Densys) [2] from the Epidemiology department of Western Provincial Department of Health.

\section{Ethical Considerations}

Ethics approval was obtained by the ethics review committee of the University of Colombo (EC-18-051) and the positive results of the Dengue antigen tests were entered to the system after prior informed written consent from the relevant parties. The patients data were kept confidently and not analysed on an individual basis.

\section{Results}

There were 401 antigen positive notified cases (54.6\% were females) to the system (Table 1 ) from 7 leading private laboratories which accounts for $18.3 \%$ from total routinely notified patients from the area within the period. Patients from 14 Medical Officer of Health (MOH) areas out of 15 were captured (Figure1). Four MOH areas accounted for the majority (57.4\%) of cases (Table 2). The percentages from the totals of 4 respective $\mathrm{MOH}$ division with weekly return of communicable diseases (WRCD) from routine surveillance and sentinel surveillance were $27.7 \%$ and $19.0 \%$ respectively.

\section{Outbreak signals}

The Dehiwala MOH area has received the majority of NS1 notifications (16.5\%) where it has reported maximum at the $28^{\text {th }}$ week (163 notifications) and $27^{\text {th }}$ week (102 confirmed cases) by sentinel site surveillance and national routine surveillance (WRCD) (Table 3) (Figure 2). In NS1 notifications, the peak has shown as a weak signal at the $24^{\text {th }}$ week and well before the $27^{\text {th }}$ week where there is a 3 week window for the preventive actions to minimise the spread of the disease. The ascending trend for NS1 Reporting in Dehiwala has started from $21^{\text {st }}$ week where it is started in WRCD after $22^{\text {nd }}$ week and sentinel surveillance after the $20^{\text {th }}$ week. In Nugegoda $\mathrm{MOH}$ area there no identifiable ascending trend with WRCD but it was evident with sentinel site surveillance after $20^{\text {th }}$ week and in NS1 notifications a weak signal spike was evident after the $20^{\text {th }}$ week (Figure 2). Two ascending trends with WRCD was noted in Maharagama $\mathrm{MOH}$ area during the $18^{\text {th }}$ and $23^{\text {rd }}$ weeks (Figure 2), but in sentinel surveillance only one ascending trend noted during the $22^{\text {nd }}$ week. NS1 reports the $23^{\text {rd }}$ week was shown the ascending trend. Pitakotte has shown the ascending trend during the $27^{\text {th }}$ week with WRCD, during the $23^{\text {rd }}$ week with sentinel surveillance and during $24^{\text {th }}$ week in the NS1 reporting system (Figure 2). In other reported areas also shows weaker signals or no outbreak signals with NS1 reporting system where the reported numbers are low.

\section{Prompt preventive actions}

Only 11 (2.7\%) cases marked as unidentified and 27 (6.7\%) cases remained as pending action in the system data. Almost all attended patients have given health advice on awareness, prevention and source reduction through premise inspection by a trained field staff with $24 \mathrm{hrs}$ of notification. In addition indoor fumigation activities also conducted in $51 \%$ of attended index premises.

Table 1. Proportion of notified NS1 positive patients from different laboratories

\begin{tabular}{ccc}
\hline Lab ID & Notified & $\%$ \\
\hline Lab 01 & 160 & 39.90 \\
Lab 02 & 80 & 19.95 \\
Lab 03 & 91 & 22.69 \\
Lab 04 & 43 & 10.72 \\
Lab 05 & 8 & 2.00 \\
Lab 06 & 14 & 3.49 \\
Lab 07 & 5 & 1.25 \\
\hline Total & $\mathbf{4 0 1}$ & $\mathbf{1 0 0 . 0 0}$ \\
\hline
\end{tabular}

Table 2. Proportion of patients reported in different $\mathrm{MOH}$ ares

\begin{tabular}{|c|c|c|c|}
\hline & MOH Area & Frequency & Percent \\
\hline 1 & MOH Dehiwala & 66 & 16.5 \\
\hline 2 & MOH Nugegoda & 61 & 15.2 \\
\hline 3 & MOH Maharagama & 55 & 13.7 \\
\hline 4 & MOH Pitakotte & 48 & 12.0 \\
\hline 5 & MOH Ratmalana & 33 & 8.2 \\
\hline 6 & MOH Battaramulla & 29 & 7.2 \\
\hline 7 & MOH Homagama & 24 & 6.0 \\
\hline 8 & MOH Kaduwela & 17 & 4.2 \\
\hline 9 & $\mathrm{MOH}$ Moratuwa & 17 & 4.2 \\
\hline 10 & MOH Boralesgamuwa & 15 & 3.7 \\
\hline 11 & MOH Kolonnnawa & 15 & 3.7 \\
\hline 12 & MOH Piliyandala & 12 & 3.0 \\
\hline 13 & MOH Kahathuduwa & 7 & 1.7 \\
\hline \multirow[t]{2}{*}{14} & MOH Padukka & 2 & 0.5 \\
\hline & Total & 401 & 100.0 \\
\hline
\end{tabular}


Table 3. Comparison of seasonal peak number of cases and reported weeks from routine national surveillance, sentinel site surveillance and NS1 surveillance in top $4 \mathrm{MOH}$ areas

\begin{tabular}{lccc}
\hline MOH Area & $\begin{array}{c}\text { WRCD Seasonal } \\
\text { Peak }\end{array}$ & $\begin{array}{c}\text { DenSys } \\
\text { Seasonal Peak }\end{array}$ & NS1 Peak \\
\hline Dehiwala & 28 (102 Cases) & 27 (163 Cases) & 24 (16 Cases) \\
Nugegoda & 32 (53 Cases) & 28 (152 Cases) & 24 (15 Cases) \\
Maharagama & 27 (62 Cases) & 27 (47 Cases) & 24 (11 Cases) \\
Pitakotte & 29 (53 Cases) & 27 (50 Cases) & 25 (12 Cases)
\end{tabular}

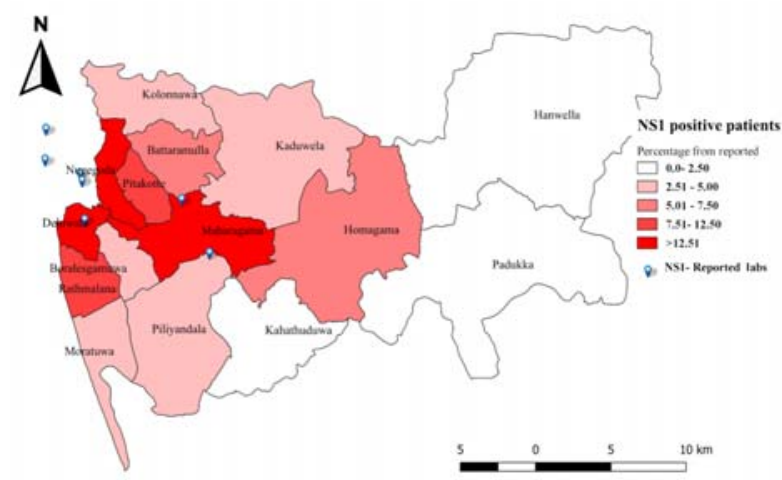

Figure 1. Relative proportion of NS1 positive cases reported in the district during $15^{\text {th }}-24^{\text {th }}$ weeks of 2016 and locations of the labs.
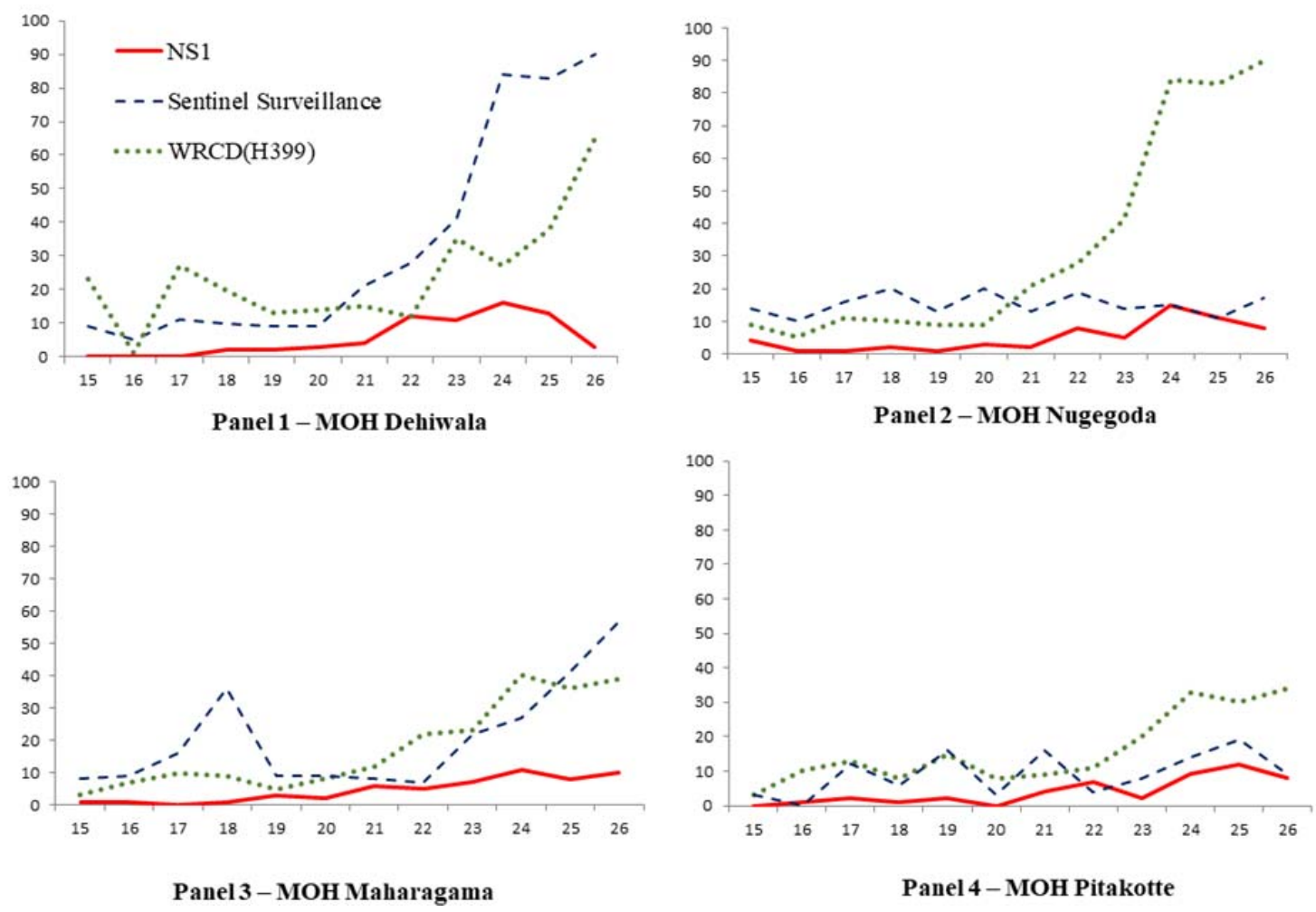

Figure 2. Comparisons of WRCD, sentinel surveillance and NS1 reporting during $15^{\text {th }}-26^{\text {th }}$ weeks of 2016 in Dehiwala, Nugegoda and Pitakotte Medical Officer of Health divisions.

\section{Discussion}

This pilot study provides an example for real-time biosurveillance for Dengue by notifying the antigen positives or true disease positives to the relevant authorities and make them to perform proactive preventive activities at the origin of the communicable disease. The model has shown promising results as an evolving EWARS which is a future requirement for the country and also it proves that the early preventive measure upon rapid notification and proper resource management is a reality of Sri Lankan context. The magnitude of the warning signals of an outbreak need to be improved by the help of various stakeholders.

The Sri Lankan surveillance system for communicable disease lacking the laboratory surveillance component for many diseases where it has been identified as a well known 
drawback within a well established time tested surveillance system. A real time variant of online laboratory initiated NS1 surveillance for Dengue (Laboratory-initiated infectious disease notifications) can be the starting point of such a system due to the impact form morbidity, mortality and public health burden from this disease. Since there is no formal regional systems of emergency response or early warning and response (EWARS) to mitigate Dengue outbreaks throughout the country. This process can be streamlined as EWARS as well. It has considered the syndromic surveillance is the leading surveillance system of many dengue epidemic countries. It has many drawbacks like lacking sensitivity and specificity [6]. This system will address most of the attributes described in a useful surveillance system described in Silvia RungeRanzinger et al in 2008 [19] such as sensitivity (proportion of outbreaks detected out of all outbreaks), timeliness (speed between outbreak detection, reporting and response), stability (ability to collect, manage and provide data properly), simplicity (ease of operation of the surveillance system), flexibility (ability to adapt during an epidemic and applicability in other settings), data quality (completeness and validity of the data recorded), representativeness (ability to describe spatial and temporal dengue distribution in the whole population, acceptability (willingness of persons/organizations to participate), accuracy/specificity (ability to distinguish between a dengue outbreak and another outbreak, between a dengue case and another illness, and between dengue haemorrhagic fever (DHF) and dengue fever and Positive Predictive Value (PPV, proportion of reported dengue cases with confirmed dengue). Merging of more than 2 scientific fields also can result better surveillance outcomes[20].

\section{Improving the capture and sensitivity}

This system was implemented in few leading private laboratories and not with the public sector hospital laboratories, where they were not performing the testing routing basis. Incorporating the majority of the available laboratories after standardizing the procedure will increase the number of data points of the system, hence increase the gross reporting as well as the magnitude of the outbreak signals from the systems towards more defined geographical area within an $\mathrm{MOH}$ areas.

In addition, its mandatory to recommend the optimised method of testing and day of testing according the current evidence and economic profile of the population to achieve the maximum yield. The genomic testing $[10,11,21,22]$ can not be recommended at large scale and it should be clearly mentioned when to do the ELISA with the sensitivity 85.6-95.9\% [23-25] or rapid testing (with or without combining IgM testing) where the sensitivity of testing varies from $60-80 \%$ [9,10,26-28]. Possibility of non invasive testing (i.e.urine, saliva) also might lead to higher compliance of testing by the public and the methods also should be evaluated for future improvements [27]. Implementation of minimum standards and guidelines for laboratories (i.e.provide the test strip with rapid test reports as a proof of testing, indications for repeated testing, etc.) will be challenging in Sri Lankan setup.

\section{Improving response}

Immediate response to a true signal is highly required because it is more precise than syndromic surveillance [29] signal and its timeliness [30]. The effective control can be achieved by mitigating the alerts and mobilising the field staff accordingly. Maintaining centrally dedicated staff for preventive measures or during the seasonal or outbreak times will be cost effective eneviour to the health department.

\section{Drawbacks in a resource poor settings}

Tendency of doing more NS1 test and insisting on admissions where there is no indications may lead to unnecessary overcrowding of wards in resource poor settings. During secondary and subsequent infections, the NS1 positivity may be reduced and the patients might end up with false assurance that they are free from the disease might lead to readmit with complicated stage and the health system may need to spend additional resources due to this or may end up with worse consequences.

\section{Limitations}

The NS1 testing was available in the private sector laboratories and it was not performed in the Government sector hospital laboratories where majority of people get admitted. The cost for the test (15-20 U.S.D.) was high compared with other available blood tests, so the majority of the patients get admitted to the government hospitals without doing the NS1 testing at the private sector. Limited number of private sector laboratories (07) involved during the pilot and three of them were situated out of the regional directorate area (Colombo Municipal Council Area) where the more patients may have tested belongs to that area might have end up with low number of cases. Most of the small laboratories also has the ability to perform the NS rapid test , there is no way to trance those availability of the facility due to limited records in the Sri Lankan health system. Ways to motivate to the laboratory staff to enter the data to the system were limited. There is not such regulatory power for the provincial health department to make legislations or make it mandatory. Continuous follow up was needed to get the data to the system by the provincial health staff. In addition there were not communication module built to send the positive cases automatically to the system without double entering due to different standards and systems used by different laboratories where it might be a burden for the laboratory staff. Since there is no mobile application developed for the system, users needed to log in by a personal computer or other device via web browser to update the system which may be a reason for the delay in updation. 
The sensitivity will change depending on the method used to detect NS1 positivity. Rapid test usually has lower value than ELISA where RT-PCR considered as the gold standard [26]. Cocirculation of other flaviviruses may lowers the specificity of NS1 test [31]. Maximizing sensitivity and specificity will be also a major concern when it comes to scaling up to National level implementation.

Finally, the coordination and support by the central epidemiology unit of the Ministry of Health is a must for the sustainability of the project where they should not consider this system as an alternative system for the national surveillance.

\section{Conclusions}

Since Dengue in Sri Lanka as well as in other countries will continue to remain during the next few decades[32] the detection of early outbreak will be critically important for prevention of outbreaks as well as minimise the intensity of them. The way forward will be a private and public laboratory integrated national realtime Dengue NS1 antigen surveillance and rapid response mechanism and the current platform is a good example of how to perform it in an effective way. The novel system can be implemented with improvements at national level would provide a better early warning and response system for Dengue in Sri Lanka and augment the existing surveillance through the directives of the ministry of health

\section{Way Forward}

This model EWARS could be extended to a supporting part of the outbreak investigation aspect as well by leading to GIS mapping, specific serotype detection, developing spatial serotype distribution mapping, identifying genotype variability beyond serotyping (i.e.phylogenetic analysis) and match with outbreak potent variants of them for effective mobilisation of field resources like in Singapore, island wide virus surveillance programme aimed at determining the serotypes and genotypes of circulating virus strains (31) with the use of national reference laboratory for Dengue or Medical Research Institute (MRI) of Sri Lanka. This can be further supported by implementing policies to strengthen the current strategies like notifying clinically-suspected and laboratory-confirmed cases within 24 hours to the relevant authorities by the laboratories.

\section{Declarations \\ Ethical Considerations}

Ethics approval was obtained by the Ethics Review Committee of the University of Colombo (EC-18-051) and the positive results of the Dengue antigen tests were entered to the system after prior informed written consent from the relevant parties. The patients data were kept confidently and not analysed on an individual basis.

\section{Availability of Data and Material}

The datasets generated during and/or analysed during the current study are not publicly available but are available from the corresponding author on reasonable request.

\section{Conflicts of interest}

No financial and non-financial competing interests to declare.

\section{Funding}

No formal funding or grants have been received. Founded by the investigators. System was developed by the $1^{\text {st }}$ author, hosted at the department server and maintained by the department staff. Pilot project was assisted by Western Provincial Department of Health, Sri Lanka providing sufficient resources.

\section{Author contributions}

All authors have contributed to the background, design, and drafting of the study and the manuscript.

\section{Acknowledgements}

Authors like to acknowledge all the administrators and all the categories of staff members of regional directorate of health, Colombo district office, provincial directorate office of Western Province office for the central assistance, encouragements, criticisms and approvals for the project, all the patients who gave the consent for disseminating the antigen testing results along with their personal details and all the public health staff under the purview of Colombo regional directorate of health. In addition, authors like to acknowledge informal financial assistance by providing necessary physical and human resources by the Western Provincial Department of Health and Colombo regional directorate of health offices.

\author{
Abbrevations \\ CMC - Colombo Municipal Council \\ CSS - Cascaded Style Sheets \\ DENV - Dengue Virus \\ DF - Dengue Fever \\ DHF - Dengue Haemorrhagic Fever \\ ELISA - Enzyme-Linked Immunosorbent Assay \\ EWARS - Early Warning and Response System \\ EWRS - Early Warning and Response System \\ GPL - General Public Licencse \\ HTML - Hypertext Markup Language \\ ICT - Information and Communication Technology
}


MOH - Medical Officer of Health

MRI - Medical Research Institute

NS1 - Non Structural Protein 1

PDHS - Provincial Director of Health Services

PPV - Positive Predictive Value

RDHS - Regional Director of Health Services

RT-PCR - Real-Time Polymerase Chain Reaction

SPSS - Statistical Package for Social Sciences

USD - United States Dollars

WRCD - Weekly Return of Communicable Diseases

\section{References}

1. Tissera HA, Jayamanne BDW, Raut R, Janaki SMD, Tozan Y, Samaraweera PC, et al. Severe Dengue Epidemic, Sri Lanka, 2017. Emerg Infect Dis [Internet]. 2020 Apr [cited 2020 Dec 27]; 26(4): 682-91. Available from: http:// wwwnc.cdc.gov/eid/article/26/4/19-0435_article.htm

2. Epidemiology Unit, Sri Lanka. National sentinel site surveillance for Dengue [Internet]. Colombo, Sri Lanka; [cited 2018 Feb 17]. Available from: http://dengue. epid.gov.lk/login.php

3. Tissa Vitarana, WS Jayakuru, Nalini Withane. Historical account of dengue haemorrhagic fever in Sri Lanka. Dengue Bull. 1997; 21(1997): 117-8.

4. Medical Research Institute, Sri Lanka. Current Dengue status in the country [Internet]. Medical Research Institute, Sri Lanka. [cited 2018 Jun 18]. Available from: http:// www.mri.gov.lk/departments/virology/dengue/

5. Sirisena PDNN, Noordeen F. Evolution of dengue in Sri Lanka-changes in the virus, vector, and climate. Int J Infect Dis. 2014; 19: 6-12.

6. Tissera H, Amarasinghe A, Gunasena S, De Silva AD, Yee LW, Sessions O, et al. Laboratory-Enhanced Dengue Sentinel Surveillance in Colombo District, Sri Lanka: 2012-2014. Bingham A, editor. PLoS Negl Trop Dis. 2016; 10(2): e0004477.

7. Wickramasinghe WM a. SB, Premaratne RG, Arunatileka SMKD. An ICT solution for issues in communicable disease surveillance in Sri Lanka. Sri Lanka J Bio-Med Inform. 2011; 1(0): S3.

8. Kosasih H, Alisjahbana B, Widjaja S, Nurhayati, Mast Q de, Parwati I, et al. The Diagnostic and Prognostic Value of Dengue Non-Structural 1 Antigen Detection in a HyperEndemic Region in Indonesia. PLOS ONE 2013; 8(11): e80891.

9. Zhang H, Li W, Wang J, Peng H, Che X, Chen X, et al. NS1-based tests with diagnostic utility for confirming dengue infection: a meta-analysis. Int J Infect Dis. 2014; 26: 57-66.

10. Aryati A, Trimarsanto H, Yohan B, Wardhani P, Fahri S, Sasmono RT. Performance of commercial dengue NS1 ELISA and molecular analysis of NS1 gene of dengue viruses obtained during surveillance in Indonesia. BMC Infect Dis [Internet]. 2013 Dec [cited 2018 Jan 2]; 13(1). Available from: http://bmcinfectdis.biomedcentral.com/articles/ 10.1186/1471-2334-13-611

11. Huhtamo E, Hasu E, Uzcátegui NY, Erra E, Nikkari S, Kantele A, et al. Early diagnosis of dengue in travelers: Comparison of a novel real-time RT-PCR, NS1 antigen detection and serology. J Clin Virol. 201; 47(1): 49-53.

12. PHP: Hypertext Preprocessor [Internet]. [cited 2019 Feb 19]. Available from: http://php.net/

13. MySQL [Internet]. [cited 2019 Feb 19]. Available from: https://www.mysql.com/

14. Cascading Style Sheets [Internet]. [cited 2019 Feb 19]. Available from: https://www.w3.org/Style/CSS/Overview. en.html

15. HTML.COM [Internet]. HTML Offical Site. Available from: https://html.com

16. GNU General Public License | Open Source Initiative [Internet]. [cited 2019 Feb 19]. Available from: https:// opensource.org/licenses/gpl-license

17. The first single application for the entire DevOps lifecycle [Internet]. GitLab. [cited 2019 Feb 19]. Available from: https://about.gitlab.com/

18. Ranwala RADLMK, Wedisinghe WAY, Perera SK. Introduction of a new laboratory based e-surveillance system for dengue infection within the Western Province, Sri Lanka. In: Commonwealth Medical Association $-24^{\text {th }}$ Triennial Conference. Colombo, Sri Lanka: CMA; 2016.

19. Runge-Ranzinger S, Horstick O, Marx M, Kroeger A. What does dengue disease surveillance contribute to predicting and detecting outbreaks and describing trends? Trop Med Int Health. 2008; 13(8): 1022-41.

20. Vitali Sintchenko, Blanca Gallego. Laboratory-guided detection of disease outbreaks: three generations of surveillance systems. Arch Pathol Lab Med. 2009; 133(6): 916-25.

21. Lai YL, Chung YK, Tan HC, Yap HF, Yap G, Ooi EE, et al. Cost-Effective Real-Time Reverse Transcriptase PCR (RT-PCR) To Screen for Dengue Virus followed by Rapid Single-Tube Multiplex RT-PCR for Serotyping of the Virus. J Clin Microbiol. 2007; 45(3): 935-41.

22. Solanke. Early dengue diagnosis: Role of rapid NS1 antigen, NS1 early ELISA, and PCR assay [Internet]. [cited 2019 Feb 23]. Available from: http://www.tjmrjournal.org/ article. asp?issn=1119-0388; year=2015; volume =18; issue $=2$; spage $=95$; epage $=99$; aulast $=$ Solanke

23. Pal S, Dauner AL, Mitra I, Forshey BM, Garcia P, Morrison AC, et al. Evaluation of Dengue NS1 Antigen Rapid Tests and ELISA Kits Using Clinical Samples. PLOS ONE [Internet]. 2014 Nov 20 [cited 2019 Feb 23]; 9(11). Available from: https://www.ncbi.nlm.nih.gov/pmc/articles/ PMC4239072/ 
24. Kumarasamy V, Wahab AHA, Chua SK, Hassan Z, Chem YK, Mohamad M, et al. Evaluation of a commercial dengue NS1 antigen-capture ELISA for laboratory diagnosis of acute dengue virus infection. J Virol Methods. 2007; 140(1-2): 75-9.

25. Ty Hang V, Minh Nguyet N, The Trung D, Tricou V, Yoksan S, Minh Dung N, et al. Diagnostic Accuracy of NS1 ELISA and Lateral Flow Rapid Tests for Dengue Sensitivity, Specificity and Relationship to Viraemia and Antibody Responses. Halstead SB, editor. PLoS Negl Trop Dis. 2009; 3(1): e360.

26. Shih HI, Hsu HC, Wu CJ, Lin CH, Chang CM, Tu YF, et al. Applications of a Rapid and Sensitive Dengue DUO Rapid Immunochromatographic Test Kit as a Diagnostic Strategy during a Dengue Type 2 Epidemic in an Urban City. PLOS ONE. 2016; 11(7): e0158437.

27. Andries AC, Duong V, Ong S, Ros S, Sakuntabhai A, Horwood P, et al. Evaluation of the performances of six commercial kits designed for dengue NS1 and anti-dengue IgM, IgG and IgA detection in urine and saliva clinical specimens. BMC Infect Dis. 2016; 16(1): 201.
28. Costa VG da, Marques-Silva AC, Moreli ML. A MetaAnalysis of the Diagnostic Accuracy of Two Commercial NS1 Antigen ELISA Tests for Early Dengue Virus Detection. Fernandez-Sesma A, editor. PLoS ONE. 2014; 9(4): e94655.

29. Meynard JB, Ardillon V, Dussart P, Chaud P, Rosine J, Durquety E, et al. Implementation of a new syndromic surveillance system in April 2006 in French Guiana. Adv Dis Surveill. 2007; 2: 159.

30. Meynard JB, Chaudet H, Green AD, Jefferson HL, Texier $\mathrm{G}$, Webber D, et al. Proposal of a framework for evaluating military surveillance systems for early detection of outbreaks on duty areas. BMC Public Health. 2008; 8(1): 146.

31. Matheus S, Boukhari R, Labeau B, Ernault V, Bremand L, Kazanji M, et al. Specificity of Dengue NS1 Antigen in Differential Diagnosis of Dengue and Zika Virus Infection. Emerg Infect Dis. 2016; 22(9): 1691-3.

32. Messina JP, Brady OJ, Pigott DM, Golding N, Kraemer MUG, Scott TW, et al. The many projected futures of dengue. Nat Rev Microbiol. 2015; 13(4): 230-9. 